PROCEEDINGS OF THE

AMERICAN MATHEMATICAL SOCIETY

Volume 128, Number 12, Pages 3671-3674

S 0002-9939(00)05544-1

Article electronically published on June 7, 2000

\title{
THE UNDECIDABILITY OF CYCLOTOMIC TOWERS
}

\author{
CARLOS R. VIDELA \\ (Communicated by Carl G. Jockusch, Jr.)
}

\begin{abstract}
Let $\mathbb{Q}\left(p^{\infty}\right)$ be the field obtained by adjoining to $\mathbb{Q}$ all $p$-power roots of unity where $p$ is a prime number. We prove that the theory of $\mathbb{Q}\left(p^{\infty}\right)$ is undecidable.
\end{abstract}

\section{INTRODUCTION}

Our main result is the undecidability of the field $\mathbb{Q}\left(p^{\infty}\right)$ in the vocabulary $L=$ $\{+, \cdot, 0,1\}$. In some cases we obtain the undecidability of the field obtained by adjoining to the rationals all $p$-power roots of unity for $p$ in a finite set of prime numbers. This is a small part of the maximal abelian extension of $\mathbb{Q}$. The decision problem for this field is open and I believe it was first raised by A. Robinson. To prove the theorem we need some results from logic and number theory explained below. Basically, a result of J. Robinson giving a condition for undecidability of algebraic integer rings, a result of ours on the definability of such rings in algebraic fields and a result of D. Rohrlich about points on elliptic curves in cyclotomic towers.

\section{Preliminary Results}

2.1. Let $R \subset \widetilde{\mathbb{Z}}$ be a ring of algebraic integers. To a formula $\varphi(x, \vec{y})$ (where $\left.\vec{y}=\left(y_{1}, \ldots, y_{n}\right)\right)$ in the vocabulary $L$ we can define a family $\left\{\varphi(x, \vec{r}): \vec{r} \in R^{n}\right\}$ of subsets of $R$ where $\varphi(x, \vec{r})=\{s \in R: R \models \varphi(s, \vec{r})\}$. The following result holds:

2.2. Proposition. Let $R \subset \widetilde{\mathbb{Z}}$ be a ring and suppose there is a collection of subsets of $R$ as above containing finite sets of arbitrarily large size. Then the ring $R$ is undecidable.

The proof of the above proposition in the case in which all sets in the family are finite and $R$ is the ring of integers of a field of algebraic numbers is due to J. Robinson [2, p. 302. It has been noted by W. Henson ([1, p. 199) that the assumption of finiteness of all sets can be dropped and it is easy to see that $R$ can be taken to be any subring of $\widetilde{\mathbb{Z}}$.

In [6] we showed that the ring $\widetilde{\mathbb{Z}} \cap \mathbb{Q}\left(p^{\infty}\right)$ is definable with parameters. That is, there exists a formula $\varphi\left(x, y_{1}, \ldots, y_{m}\right)$ in $L$ such that for some $\alpha_{1}, \ldots, \alpha_{m} \in \mathbb{Q}\left(p^{\infty}\right)$ we have

$$
r \in \widetilde{\mathbb{Z}} \cap \mathbb{Q}\left(p^{\infty}\right) \Longleftrightarrow \mathbb{Q}\left(p^{\infty}\right) \models \varphi\left(r, \alpha_{1}, \ldots, \alpha_{m}\right) .
$$

Received by the editors November 23, 1998 and, in revised form, February 1, 1999.

1991 Mathematics Subject Classification. Primary 03B25, 12L05.

(C)2000 American Mathematical Society 
The set of parameters $\left\{\alpha_{1}, \ldots, \alpha_{m}\right\}$ is troublesome in what follows, so we work with the ring $R_{p}$ defined below which is definable in $L$ :

$r \in R_{p}$ if and only if $\forall c_{1} \ldots c_{m} \in \mathbb{Q}\left(p^{\infty}\right)$ (if $\varphi\left(x, c_{1}, \ldots, c_{m}\right.$ ) is a ring $\Rightarrow$ $\left.\varphi\left(r, c_{1}, \ldots, c_{m}\right)\right)$.

By the above result $\mathbb{Z} \subset R_{p} \subset \widetilde{\mathbb{Z}} \cap \mathbb{Q}\left(p^{\infty}\right)$. Hence to establish the undecidability of $\mathbb{Q}\left(p^{\infty}\right)$ it is enough to show that Proposition 2.2 holds for $R_{p}$. In order to prove this we will use a result of D. Rohrlich ([3], p. 409) stated in 2.3 below.

As was pointed by L. van den Dries, the use of $R_{p}$ is not necessary. It turns out that $\widetilde{\mathbb{Z}} \cap \mathbb{Q}\left(p^{\infty}\right)$ is already definable without parameters. The argument is as follows. Let $\mathbb{Q}\left(\alpha_{1}, \ldots, \alpha_{m}\right)=\mathbb{Q}(\alpha)$ and let $f$ be the minimal polynomial of $\alpha$ over $\mathbb{Q}$. Let $\alpha_{i}=g_{i}(\alpha)$ with $g_{i} \in \mathbb{Q}[x]$ and the degree of $g_{i}$ less than the degree of $f$. In the formula above (defining $\widetilde{\mathbb{Z}} \cap \mathbb{Q}\left(p^{\infty}\right)$ ) replace each occurrence of $\alpha_{i}$ by $g_{i}(\alpha)$ and add the condition $f(\alpha)=0$. This gives us a new formula $\phi(x, \alpha)$ which defines in $\mathbb{Q}\left(p^{\infty}\right)$ the ring $\widetilde{\mathbb{Z}} \cap \mathbb{Q}\left(p^{\infty}\right)$. Since $\mathbb{Q}\left(p^{\infty}\right)$ and $\widetilde{\mathbb{Z}} \cap \mathbb{Q}\left(p^{\infty}\right)$ are closed under conjugation one can eliminate the occurrence of $\alpha$ by quantifying it out.

2.3. Let $E$ be an elliptic curve defined over $\mathbb{Q}$ with complex multiplication by the ring of integers of an imaginary quadratic field, and let $P$ be a finite set of primes where $E$ has good reduction. Let $L$ be the maximal abelian extension of $\mathbb{Q}$ unramified outside $P$ and infinity and let $E(L)$ be the group of points on $E$ which are defined over $L$. Then $E(L)$ is finitely generated.

\section{Main Results}

3.1. We first consider $p$ odd. Let $E$ be the elliptic curve $y^{2}=x^{3}+8 x$. It has discriminant equal to $-2^{15}, j$-invariant equal to $(12)^{3}$ and has complex multiplication by the ring of integers of $\mathbb{Q}(\sqrt{-1})$. The point $P_{0}=(1,3)$ belongs to $E(\mathbb{Q})$ and has infinite order since $2 P_{0}=\left(\frac{7^{2}}{6^{2}}, \frac{113.7}{6^{3}}\right)$.

We will use $E$ to define a family of sets in $\mathbb{Q}\left(p^{\infty}\right)$ as required in Proposition 2.2 First note the following.

Suppose $\left(\frac{a}{b}, \frac{c}{d}\right) \in E(\mathbb{Q})$ with $a, b, c, d \in \mathbb{Z},(a, b)=(c, d)=1$. Then we may assume $b^{3}=d^{2}$ because from the equation it follows that $b^{3} c^{2}=d^{2}\left(a^{3}+8 a b^{2}\right)$ and so $b^{3} \mid d^{2}$ and $d^{2} \mid b^{3}$. Hence $d^{2}= \pm b^{3}$. We may take $b>0$ since if $b<0$, then $b=-b^{\prime}$ with $b^{\prime}>0$ and $\frac{a}{b}=\frac{-a}{b^{\prime}}$.

3.2. For the next step, take $N$ points $P_{i}=\left(\frac{a_{i}}{b_{i}}, \frac{c_{i}}{d_{i}}\right) \in E(\mathbb{Q}) a_{i}, b_{i}, c_{i}, d_{i} \in \mathbb{Z}$, $\left(a_{i}, b_{i}\right)=1$. We twist $E$ to a new curve which has $N$ integral points. Here we follow the idea involved in exercise 9.3 of Silverman's book [4], p. 272.

Let $E_{\bar{b}}$ be the curve $y^{2}=x^{3}+8\left(b_{1} \cdots b_{N}\right)^{2} x$. Then the integral set of points $P_{i}=$ $\left(a_{i} b_{1} \cdots b_{i-1} b_{i+1} \cdots b_{N}, c_{i} d_{1} \cdots d_{i-1} d_{i+1} \cdots d_{N}\right)$, for $1 \leq i \leq N$ belong to $E_{\bar{b}}$ as is easily checked. On the other hand the discriminant of $E_{\bar{b}}$ is equal to $-2^{15}\left(b_{1} \cdots b_{N}\right)^{6}$ and the $j$-invariant is $12^{3}$. Hence $E_{\bar{b}}$ also has complex multiplication by the ring of integers of $\mathbb{Q}(\sqrt{-1})$.

3.3. Let $p$ be an odd prime, and let $n_{p}$ be the size of the group $E\left(\mathbb{F}_{p}\right)$. Choose $\ell$ a prime number bigger than $n_{p}$. Then the sequence of points $P_{0}, \ell P_{0}, \ell^{2} P_{0}, \ldots$ is infinite and if $\ell^{n} P_{0}=\left(\frac{a_{n}}{b_{n}}, \frac{c_{n}}{d_{n}}\right)$ with $a_{n}, b_{n}, c_{n}, d_{n} \in \mathbb{Z} \quad\left(a_{n}, b_{n}\right)=\left(c_{n}, d_{n}\right)=1$ we have:

a) $\widetilde{P_{0}} \neq \widetilde{0}($ here $\sim$ is reduction $\bmod p)$,

b) $\widetilde{\ell^{n} P_{0}}=\ell^{n} \widetilde{P_{0}} \neq \widetilde{0}$. 
Part a) is obvious. To see b) note that the order of $P_{0}$ in $E\left(\mathbb{F}_{p}\right)$ is bigger than one and divides $n_{p}$. If $\ell^{n} \widetilde{P_{0}}=\widetilde{0}$, then $n \geq 1$ and $\operatorname{order}\left(P_{0}\right) \mid \ell^{n}$ which is impossible.

Now $\ell^{n} \widetilde{P_{0}} \neq \widetilde{0}$ implies that $b_{n} \not \equiv 0 \bmod p$ and $d_{n} \not \equiv 0 \bmod p$. Otherwise, if $p$ divides either $b_{n}$ or $d_{n}$, then $3 \operatorname{ord}_{p} b_{n}=2 \operatorname{ord}_{p} d_{n}>0$ and so projectively $\ell^{n} P_{0}=$ $\left[\frac{a_{n} d_{n}}{b_{n}}, c_{n}, d_{n}\right]$. Reducing $\bmod p$ we get $\ell^{n} \widetilde{P_{0}}=[0,1,0]=\widetilde{0}$ which is impossible.

We are almost done. Apply 2.3 to the curve $E_{\bar{b}}$ to conclude that $E_{\bar{b}}\left(\mathbb{Q}\left(p^{\infty}\right)\right)$ is finitely generated. Hence all the points are contained in a finite extension $L_{n}$ of $\mathbb{Q}$. By Siegel's theorem the set of integral points of $E_{\bar{b}}\left(L_{n}\right)$ is finite and by combining 3.2 and 3.3 we can make this finite set arbitrarily large.

We have therefore established Proposition 2.2 for $R_{p}$. As our definable collection of sets we can use $\left\{\exists x\left(y^{2}-x^{3}+8 \lambda x=0\right): \lambda \in R_{p}\right\}$.

3.4. Let $p=2$. For this case we use $E: y^{2}+y=x^{3}-38 x+90$. The discriminant of $E$ is $-19^{3}$ and $j=-3^{3} \cdot 2^{15}$. The curve has complex multiplication by the ring of integers of $\mathbb{Q}(\sqrt{-19})([\sqrt[5]{ }$, p. 483$)$.

The point $P=(0,9) \in E(\mathbb{Q})$ and $2 P=(4,-2), 4 P=\left(\frac{28}{9}, \frac{-53}{27}\right)$.

Hence $P$ has infinite order. If $\left(\frac{a}{b}, \frac{c}{d}\right) \in E(\mathbb{Q})$ (with $a, b, c, d \in \mathbb{Z},(a, b)=(c, d)=$ $1)$, then as before we may assume $d^{2}=b^{3}$. To get curves from $E$ which have integral points and satisfy 2.3 as in the odd case we use $E_{d, b}: y^{2}+d y=x^{3}-38 b^{2} x+90 b^{3}$ with $d^{2}=b^{3}$. A calculation shows that the discriminant of $E_{d, b}$ is $-19^{3} b^{6}$ and the $j$ invariant is $-3^{3} \cdot 2^{15}$. We may now repeat the argument in 3.3

3.5. As a final remark note the following. Let $A$ be a finite set of prime numbers and define $\mathbb{Q}_{A}$ to be the field obtained by adjoining to $\mathbb{Q}$ all $p$-power roots of unity to $\mathbb{Q}$ for $p \in A$. In [6] we showed that $\widetilde{\mathbb{Z}} \cap \mathbb{Q}_{A}$ is definable. So, for certain finite sets $A$ of prime numbers we obtain the undecidability of $\mathbb{Q}_{A}$. For example, if the set $A$ consists of odd primes, then we may use the construction in 3.1 3.3. For an arbitrary finite set of primes we only have a partial result. First we need a lemma of D. Rohrlich:

Lemma. Let $A$ be a finite set of primes. Then there exists an elliptic curve $E$ over $\mathbb{Q}$ and a point of infinite order $Q \in E(\mathbb{Q})$ such that $E$ has good reduction at every $p \in A$ and the reduction of $Q$ modulo $p$ is nonzero for every $p \in A$.

Proof. For each $p \in A$ choose an elliptic curve $E_{p}$ over $\mathbb{F}_{p}$ and a nonzero point $Q \in E_{p}\left(\mathbb{F}_{p}\right)$. For $p \geq 5$ the existence of a nonzero point in $E_{p}\left(\mathbb{F}_{p}\right)$ is automatic for any $E_{p}$. For $p=2$ or 3 we can choose $E_{p}$ so that $E_{p}\left(\mathbb{F}_{p}\right) \neq\{0\}$. Since $A$ can be enlarged without loss of generality, we may assume that there are distinct primes $r, s, t \in A$ such that $2 Q_{r} \neq 0,2 Q_{s}=0$, and $2 Q_{t}=0$. Thus $Q_{s}$ and $Q_{t}$ are points of order 2 but $Q_{r}$ has order $>2$. For each $p \in A$ choose a generalized Weierstrass equation for $E_{p}$ over $\mathbb{F}_{p}$, say

$$
E_{p}: y^{2}+a_{1, p} x y+a_{3, p} y=x^{3}+a_{2, p} x^{2}+a_{4, p} x+a_{6, p},
$$

and write $Q_{p}=\left(u_{p}, v_{p}\right)$. By the Chinese Remainder Theorem there are integers $a_{1}, a_{2}, a_{3}, a_{4}, a_{5}, a_{6}, u, v$ such that $a_{i} \equiv a_{i, p}, u \equiv u_{p}$, and $v \equiv v_{p}$ modulo $p$ for all $p \in A$. Put

$$
c=\left(v^{2}+a_{1} u v+a_{3} v\right)-\left(u^{3}+a_{2} u^{2}+a_{4} u+a_{6}\right)
$$

and define $E$ by

$$
E: y^{2}+a_{1} x y+a_{3} y=x^{3}+a_{2} x^{2}+a_{4} x+\left(a_{6}+c\right) .
$$


Since $c \equiv 0$ modulo $p$ for all $p \in A$ we see that the equation for $E$ reduces modulo $p$ to the equation for $E_{p}$. In particular, since $A$ may be assumed nonempty it follows that $E$ is an elliptic curve (i.e. the discriminant of the above equation for $E$ is nonzero because it is nonzero modulo $p$ for $p \in A$ ). Also $E$ has good reduction modulo $p$ for all $p \in A$, and the point $Q=(u, v) \in E(\mathbb{Q})$ reduces to $Q_{p}$ for all $p \in A$ and in particular reduces to a nonzero point for each $p \in A$. We claim that $Q$ has infinite order. It suffices to show that $2 Q$ has infinite order. Now $2 Q$ is nonzero because it is nonzero modulo $r$. But $2 Q$ reduces to zero modulo two distinct primes, namely $s$ and $t$, and therefore, since it is nonzero, it is also not a torsion point (a nonzero torsion point can reduce to zero in at most one characteristic). Therefore $2 Q$ has infinite order.

Next, we repeat the constructions in 3.2 and 3.3 to the curve $E$ and point $Q$ of the lemma. Writing $Q=\left(\frac{a}{b}, \frac{c}{d}\right) \quad a, b, c, d \in \mathbb{Z},(a, b)=(c, d)=1$ it follows that $d^{2}=b^{3}$ and the twist we use is

$$
E_{d, b}: y^{2}+a_{1} \frac{d}{b} x y+a_{3} d y=x^{3}+a_{2} b x^{2}+a_{4} b^{2} x+\left(a_{6}+c\right) b^{3} .
$$

The discriminant of $E_{d, b}$ is $b^{6} \Delta_{E}$ and the $j$ invariant is equal to that of $E$. The argument in 3.3 fails only at the point where we apply Rohrlich's theorem. In general, we cannot expect $E$ to have complex multiplication. However, as Rohrlich points in his paper (3], p. 422), if the Taniyama-Weil and the Birch-SwinnertonDyer conjectures are true, then his theorem holds for all elliptic curves over $\mathbb{Q}$. Hence $\mathbb{Q}_{A}$ would be undecidable for all finite sets of prime numbers.

\section{REFERENCES}

[1] L. van den Dries, Elimination theory for the ring of algebraic integers, J. reine angew. Math. 388 (1988), 189-205. MR 89k:03038

[2] J. Robinson, On the decision problem for algebraic rings, Studies in Mathematical Analysis and Related Topics, Standford Univ. Press, Standford 1962, 297-304. MR 26:3609

[3] D.E. Rohrlich, On $L$-functions of elliptic curves and cyclotomic towers, Invent. Math. 75 (1984), 409-423. MR 86g:11038b

[4] J. Silverman, The arithmetic of Elliptic Curves, G.T.M. 106, Springer-Verlag, New York 1986. MR 87g:11070

[5] J. Silverman, Advanced Topics in the Arithmetic of Elliptic Curves, G.T.M 151, SpringerVerlag, New York 1994. MR 96b:11074

[6] C.R. Videla, Definability of the ring of integers in pro- $p$ extensions of numbers fields, submitted (1997).

Departamento de Matemáticas, CinVestaV-IPN, A. Postal 14-740, México, D.F. 07000, MÉXICO

E-mail address: cvidela@math.cinvestav.mx 